\title{
Implementación universal de un cribado de defectos congénitos de garantía en un área sanitaria: área hospitalaria de Valme, Sevilla, España
}

\author{
José A. Sainz B. ${ }^{1}$, Rosa Serrano R. ${ }^{1}$, Ignacio Peral M. ${ }^{2}$, Enriqueta Turmo F. ${ }^{1}$, Antonio \\ Moro 0. 2, Carmen Almeida G. 3a, Virginia Caballero F. '1, Rogelio Garrido T. '1 \\ 1 Departamento de Obstetricia y Ginecología, ${ }^{2}$ Departamento de Bioquímica, ${ }^{3}$ Unidad de Investigación. Hospital \\ Universitario Valme. Sevilla. España.
}

a Estadístico.

\section{RESUMEN}

Antecedentes: La posibilidad de que un recién nacido presente algún tipo de defecto congénito al nacimiento es de un 2-4\% y la aplicación de métodos de cribado de cromosomopatías y de malformaciones estructurales puede reducir la prevalencia de estos defectos congénitos al nacimiento. Objetivos: Demostrar que es posible la implantación de un cribado de malformaciones congénitas de garantía (sensibilidad de diagnóstico para malformaciones estructurales mayores y para síndrome de Down del $80 \%$ ) y universal (aplicado al $90 \%$ de gestantes). Proponemos que la implantación de este cribado supone una disminución de la tasa de los defectos congénitos no diagnosticados al nacimiento a menos del $0,5 \%$ de los recién nacidos. Método: Estudio prospectivo. Hemos valorado 12.478 gestantes (julio 2006-septiembre de 2009). Método de cribado de defecto congénitos: test combinado asociado a ecografía morfológica (18-22 semanas) Resultados: La prevalencia de defecto congénito fue de 2,26\% [IC 95\%: 1,9-2,5] (282/12478). Valoración ecográfica fue del $99,2 \%$ de las gestantes. Tasa de diagnóstico de malformaciones estructurales fue de $79,3 \%$ [IC $95 \%$ : 74,3-84,4] (196/247) y 95,6\% [IC 95\%: 91,8-99,3] (110/115) para las malformaciones mayores. Se ofertó un cribado de cromosomopatias al $95,1 \%$ de las gestantes con una tasa de diagnóstico del $88,5 \%$ [IC 95\%: 79,9-99] (31/35). Conclusiones: Un cribado de defectos congénitos universal y de garantías logró disminuir la prevalencia de defectos congénitos al nacimiento sin diagnosticar a un $0,5 \%$.

\section{PALABRAS CLAVE: Defectos congénitos, malforrmación estructural, síndrome de Down, cromoso- mopatías, diagnóstico prenatal}

\section{SUMMARY}

Background: The probability of a newborn presenting some kind of congenital defect at birth is $2-4 \%$ and the application of methods of screening for chromosomal and structural abnormalities can reduce the prevalence of these defects at birth. Objectives: The aim of this study is to prove that it is possible to implement a screening for congenital malformations that is standardised (diagnostic sensitivity [Sen] $>80 \%$ for major structural deformations and Down's syndrome) and universal (90\% of pregnant women). We also want to prove that this screening reduces the rate of undiagnosed congenital defects at birth. Methods: Prospective study. We assessed 12,478 pregnant women (July 2006- September 2009). A morphological ultrasound (18-22 weeks) and a combined test were carried out as the methods for screening for congenital defects. Results: The prevalence of congenital defects was 2.26\% [95\% Cl: 1.9-2.5] (282/12478). The ultrasound 
scan was performed on $99.2 \%$ of the pregnant women. There was a Sen of $79.3 \%$ [95\% Cl: $74.3-84.4]$ (196/247) for structural malformation and 95.6\% [95\% Cl: 91.8-99.3] (110/115) for major malformations). Screening for chromosomal anomalies was performed on $95.1 \%$ of pregnant women with a Sen of $88.5 \%$ [95\% Cl: 79.9-99] (31/35). Conclusions: A standardised and universal screening for congenital defects reduced the prevalence of undiagnosed congenital defects at birth to $0.5 \%$.

\section{KEY WORDS: Congenital defects, congenital malformation, Down's syndrome, chromosomal ano- malies, prenatal diagnosis}

\section{INTRODUCCIÓN}

La posibilidad de que un recién nacido presente algún tipo de defecto congénito al nacimiento es de un $2-4 \%$ (1), de estos el $60 \%$ se deben a malformaciones estructurales y el $12-15 \%$ se deben a cromosomopatías (2).

Son dos los métodos de cribado que han demostrado ser útiles en la identificación de defectos congénitos: el cribado de cromosomopatías y el cribado de malformaciones estructurales (1,3-7). Sin embargo, estos métodos de cribado se deben de aplicar correctamente para garantizar una buena sensibilidad diagnóstica (sensibilidad del $85 \%$ para una tasa de falsos positivos del $5 \%$ en el cribado de cromosomopatías y una sensibilidad para el diagnóstico de malformación estructural mayor del $60 \%$ ). Además, estos métodos de cribado se deben aplicar universalmente ya que no existen grupos de riesgo establecido para estas patologías (3).

El objetivo de esta comunicación es demostrar que es posible implantar un cribado estandarizado o de garantías y universal de anomalías cromosómicas fetales y malformaciones estructurales, en gestantes de bajo riesgo para defecto congénito, y también que la implantación de este cribado reduce la tasa de defectos congénitos sin diagnóstico al nacer a menos del $0,5 \%$.

\section{MATERIAL Y METODO}

El estudio incluye un periodo de 3 años (julio 2006-septiembre 2009), con un total de 12.478 gestaciones pertenecientes a nuestra área sanitaria, la cual está conformada por unos 356.318 habitantes.

Cribado de malformaciones estructurales. Seguimos la clasificación de malformaciones propuestas por EUROFETUS (9). La ecografía morfológica se realiza a las 20 semanas (18-22 semanas), con consentimiento informado previo y centralizado en la Unidad de Diagnóstico Prenatal del Hospital Universitario de Valme, con un tiempo medio para cada ecografía de 20 minutos y realizada por tres ecografístas con gran experiencia (más de 5 años de dedicación exclusiva en ecografía obstétrica), y dos personas con experiencia limitada (entre 1 y 3 años de dedicación exclusiva) siguiendo las recomendaciones de la Sociedad Española de Obstetricia y Ginecología (8) y del Royal College of Obstetricians and Gynaecologists (9) sobre la realización de la ecografía morfológica.

Todos los recién nacidos son controlados, por el Servicio de Pediatría del Hospital Universitario de Valme, Sevilla, España, durante los 3 primeros días tras el nacimiento. En caso de sospecha o diagnóstico postnatal de malformación, se realiza seguimiento pediátrico a los 6 meses y al año. En caso de interrupción del embarazo a los restos abortivos se les realiza estudio anatomopatológico.

Cribado de cromosomopatías. Se oferta cribado de cromosomopatías, con información previa y consentimiento informado, que consiste en el Test Combinado (pregnancy-associated plasma protein A [PAPP-A], free beta-human chorionic gonadotropin [fbHCG], Translucencia Nucal [TN]) en aquellas gestantes que acceden al control gestacional antes de las 13 semanas de gestación y en aquellos casos que el acceso es superior a las 13 semanas se les aplica un Doble Test (B-chain of human chorionic gonadotropin $[\beta-h C G]$ y Alfa Fetoproteina $[\alpha-F P])$.

Las muestras para el Test Combinado fueron obtenidas entre las $8+0$ y $12+6$ semanas de gestación y para el Doble Test entre las $14+0$ y $17+6$ semanas de gestación. En todos los casos el espécimen fue suero, que se conservo a $4^{\circ} \mathrm{C}$ hasta su procesamiento (este se produjo en un tiempo medio de 24 horas y máximo de 72 horas). Se emplea el analizador INMULITE 2000 (SIEMENS) y sus correspondientes reactivos (PAPP-A INMULITE 2000; BHCG-F INMULITE 2000; AFP INMULITE 2000). EI método utilizado para el análisis fue en todos los casos un enzimoinmuno análisis quimioluminiscente en fase sólida.

El examen ecográfico se realiza por 22 obstetras con formación en ecografía de 11-13+6 semanas para la valoración de la Translucencia Nucal (TN), y de la longitud céfalo-caudal (CRL) por vía abdominal $(5 \mathrm{MHz})$ o por vía vaginal $(8 \mathrm{MHz})$ si- 
guiendo las recomendaciones de la Fetal Medicine Foundation (10).

El software empleado para el cálculo de los índices de riesgo fue PRISCA Typolog versión 4.0. El riesgo individual de una embarazada se estima multiplicando el riesgo a priori debido a la edad materna, por la razón de verosimilitud del perfil de los marcadores empleados (PAPP-A, BHCG-L, TN en el test combinado y AFP, BHCG-L en el doble test) $(11,12)$. Los múltiplos de la mediana (MoM) se corrigen en función de factores maternos como el peso, tabaquismo, diabetes, gemelaridad, FIV y origen étnico (13). Un valor mayor o igual a 1 en 270 s para SD se informa como riesgo elevado y se oferta una técnica invasiva. En caso de síndrome de Edwars el riesgo elevado se considera aquel que el riesgo es $\geq$ a 1 en 100 . También se indica la realización de técnica invasiva ante la identificación de una malformación mayor en la evaluación ecográfica morfológica fetal (20 semanas).

Requisitos del cribado de cromosomopatias y malformaciones estructurales

De Garantía:

a. Cribado de cromosomopatías: Test Combinado consecutivo y descentralizado (realizado en la consulta básica de obstetricia), con una tasa de diagnóstico para el síndrome de Down del $80 \%$ y para todas las cromosomopatías del $85 \%$ para una tasa de falsos positivos del 5\%. En caso de incorporación al control de embarazo en el segundo trimestre, se aplicará un doble test bioquímico con una tasa de diagnóstico del $60 \%$ para el síndrome de Down para una tasa de falsos positivos del $15 \%$.

b. Cribado de malformaciones estructurales: Ecografía morfológica centralizada con una tasa de diagnóstico para las malformaciones mayores mayor del $80 \%$ y para las malformaciones menores mayor del $60 \%$.

Universal: Ambos métodos de cribado aplicados al menos al $90 \%$ de las gestantes.

Análisis estadístico. Para el cálculo del tamaño muestral, en el momento del nacimiento y tras la aplicación del cribado de defectos congénitos, para estimar una disminución esperada del $2,5 \%$ en la prevalencia de anomalías congénitas, establecida en el $3 \%$ en una población finita de 12.500 embarazadas, fijado un error $\alpha$ del $5 \%$ y una potencia del $80 \%$ en una prueba bilateral, se determinó un tamaño muestral de 150 gestantes con anomalías congénitas.

Para estimar una sensibilidad esperada del $85 \%$ frente al $75 \%$ establecida en la prueba de cribado de cromosomopatías para esa misma población, fi- jados un error $\alpha$ de 0,045 (4,5\% de falsos positivos), una prevalencia de cromosomopatías del 0,33\% (1 de 300 ) y una potencia del $80 \%$ en una prueba bilateral, resultó un tamaño muestral necesario de 34 embarazadas con cromosomopatías.

Para estimar una sensibilidad esperada del $75 \%$ frente al $65 \%$ establecida en la prueba de cribado de malformaciones estructurales mayores en dicha población, con una tasa de falsos positivos del $1 \%$, una prevalencia de malformaciones del $2 \%$ y una potencia del $80 \%$ en una prueba bilateral, el tamaño muestral necesario fue de 126 embarazadas con malformaciones.

Todos los tamaños muestrales se determinaron con el programa nQuery Advisor 4.0. El análisis de los datos se realizó con el paquete estadístico SPSS 14.0 para Windows (14).

\section{RESULTADOS}

Durante el tiempo de estudio hubo un total de 12.478 gestaciones con un número de 12.668 neonatos. En la Tabla I se describen los resultados obstétricos y perinatales del grupo de estudio; 282 fetos (2,26\%) [IC 95\%: 1,9-2,5](282/12478) presentaban algún tipo de defecto congénito con 78 casos de interrupciones del embarazo (47 por malformación estructural y 31 por cromosomopatía). Hubo 35 casos de cromosomopatías (prevalencia $0,28 \%$ ), el síndrome de Down fue el defecto cromosómico más frecuente ( 28 casos, el $80 \%$ de las cromosomopatías).

Se ofertó el cribado de cromosomopatías a 12.035 gestantes $(95,11 \%$ de la población). Se realizó un test combinado en el $86,85 \%$ de los casos (10.452 pacientes) y un doble test en el $6,72 \%$. El $6,43 \%$ de las gestantes (773 casos) revocaron el cribado. En un 1,81\% de los casos (188 gestantes) fue necesaria la reevaluación ecográfica de la TN. Se observaron 35 cromosomopatías de las cuales se han diagnosticado prenatalmente $31 \mathrm{ca}-$ sos, (88,5\%) [IC 95\%: 79,9 a 99](31/35) y 4 niños nacieron con síndrome de Down. En 2 de estos 4 casos de síndrome de Down no diagnosticado, las gestantes no han deseado el cribado de cromosomopatías.

En la Tabla II se describe la sensibilidad y tasa de falsos positivos del test combinado y la de su asociación a la ecografía morfológica para el síndrome de Down y todas las cromosomopatías. En la Tabla III se describen los casos cromosomopatías diagnosticadas y sin diagnosticar. 
Tabla I

RESULTADOS OBSTÉTRICOS EN EL HOSPITAL UNIVERSITARIO DE VALME

\begin{tabular}{lc}
\hline $\begin{array}{l}\text { Número de gestaciones } \\
\text { Número de gestaciones }\end{array}$ & 12.478 \\
gemelares & $267(2,14 \%)$ \\
$\begin{array}{l}\text { Edad media de las gestantes } \\
\text { (años) }\end{array}$ & $30,0 \pm 5,3(14-47)$ \\
$\begin{array}{l}\text { Edad gestacional media al } \\
\text { parto (semanas) }\end{array}$ & $38,97 \pm 1,6(24-42)$ \\
$\begin{array}{l}\text { a: } \text { Tasa de parto prematuro } \\
<37 \text { semanas }\end{array}$ & $a: 7,1 \%$ \\
$\begin{array}{l}\text { b: Tasa de parto prematuro } \\
<32 \text { semanas }\end{array}$ & b: $1,2 \%$ \\
$\begin{array}{l}\text { Tasa de cesárea } \\
\text { Tasa de recién nacidos con } \\
\text { peso } \leq 2500 \text { gramos }\end{array}$ & $20,5 \%$ \\
$\begin{array}{l}\text { Tasa de recién nacidos con } \\
\text { peso } \leq 1000 \text { gramos }\end{array}$ & $7,4 \%$ \\
$\begin{array}{l}\text { Número de recién nacidos } \\
\text { vivos } \\
\text { Número de recién nacidos } \\
\text { muertos } \\
\text { Número de interrupción } \\
\text { voluntaria del embarazo }\end{array}$ & $0,34 \%$ \\
\hline
\end{tabular}

En 247 fetos se presentó algún tipo de malformación estructural, prevalencia del 1,9\% [IC 95\%: 1,7-2,2]. Hubo 115 casos $(46,5 \%)$ de malformación mayor, prevalencia de 0,9\% [IC 95\%: 0,7-0,9]. Las malformaciones estructurales más frecuentes fueron las cardiopatías $(28,3 \%)$ seguida de las renales $(25,9 \%)$ y de las del sistema nervioso central $(15,7 \%)$ (Tabla IV). No fue posible realizar la ecografía morfológica en 59 casos $(0,47 \%)$ y en el $3,3 \%$ de las gestantes (412 casos) fue necesaria la repetición de esta.

La tasa de detección de malformaciones fue del 79,3\% [IC 95\%: 74,3-84,4](196 casos diagnosticados) y antes de las 24 semanas del 77,7\% [IC 95\%: 72,5-82,9](192 casos) (Tabla IV). Se diagnosticó el 95,5\% [IC 95\%: 91,8-99,3](110/115 casos) de las malformaciones congénitas mayores y el $65,1 \%$ [IC 95\%: 57-73,3](86/132) de las menores (Tabla IV). Se observaron diferencias en las tasas de diagnóstico en función del sistema anatómico fetal evaluado (Tabla V). Se observó una tasa de falsos positivos del 0,09\% [IC 95\%: 0,04-0,1] sobre todas las gestaciones (12 casos: 6 casos de la comunicación interventricular, 1 caso de comunicación interauricular, 1 sospecha de coartación de aorta, 1 caso de persistencia de vena umbilical derecha, 1 caso de sospecha de drenaje venoso anómalo y 2 casos de deformidad de las extremidades).

Tabla II

\section{SENSIBILIDAD Y TASA DE FALSOS POSITIVOS DEL TEST COMBINADO Y SU ASOCIACIÓN A LA ECOGRAFÍA MORFOLÓGICA, COMO MÉTODO DE CRIBADO DE LA TRISOMIA 21 Y OTRAS CROMOSOMOPATÍAS}

\begin{tabular}{|c|c|c|c|c|}
\hline & \multicolumn{2}{|c|}{ Para trisomía 21} & \multicolumn{2}{|c|}{ Para todas las cromosomopatías } \\
\hline & $\begin{array}{c}\text { Total de } \\
\text { gestaciones }\end{array}$ & $\begin{array}{l}\text { Gestantes que } \\
\text { desean cribado de } \\
\text { cromosomopatía }\end{array}$ & $\begin{array}{l}\text { Total de } \\
\text { gestaciones }\end{array}$ & $\begin{array}{c}\text { Gestantes que desean } \\
\text { cribado de cromoso- } \\
\text { mopatía }\end{array}$ \\
\hline $\begin{array}{l}\text { Sensibilidad del test } \\
\text { combinado }\end{array}$ & $\begin{array}{c}71,4 \%(20 / 28) \\
{[\text { IC 95\%: 54,6-88,1] }}\end{array}$ & $\begin{array}{c}76,9 \%(20 / 26) \\
\text { [IC 95\%: 60,7-93,1] }\end{array}$ & $\begin{array}{c}68,5 \%(24 / 35) \\
{[\text { IC 95\%: 53,1-83,9] }}\end{array}$ & $\begin{array}{c}72,7 \%(24 / 33) \\
{[\text { IC 95\%: 57,5-87,9] }}\end{array}$ \\
\hline $\begin{array}{l}\text { Sensibilidad del test } \\
\text { combinado asociado } \\
\text { a ecografía morfo- } \\
\text { lógica }\end{array}$ & $\begin{array}{c}85,7 \%(24 / 28) \\
{[I C \text { 95\%: 78,5-98] }}\end{array}$ & $\begin{array}{c}92,3 \%(24 / 26) \\
\text { [IC 95\%: 85,5-99,9] }\end{array}$ & $\begin{array}{c}88,5 \%(31 / 35) \\
{[\text { IC 95\%: 79,9 a 99] }}\end{array}$ & $\begin{array}{c}93,9 \%(31 / 33) \\
{[\text { IC 95\%: 85,7-99,9] }}\end{array}$ \\
\hline
\end{tabular}

Tasa de falsos positivos del test combinado: 4,3\% [IC 95\%: 3,9-4,6] (537/12.478 casos). Tasa de falsos positivos del test combinado asociado a la ecografía morfológica: 5\% (623/12478) [IC 95\%: 4,6-5,4]. 
Tabla III

DATOS DEL CRIBADO DE CROMOSOMOPATÍAS DE LOS CASOS DIAGNOSTICADOS Y NO DIAGNOSTICADOS

\begin{tabular}{|c|c|c|c|c|c|c|c|c|c|c|}
\hline$n$ & $\begin{array}{l}\text { Edad } \\
\text { (años) }\end{array}$ & $\begin{array}{l}\text { Edad } \\
\text { riesgo } \\
(1:)\end{array}$ & $\begin{array}{l}\text { ß-hCG } \\
\text { (MoM) }\end{array}$ & $\begin{array}{l}\text { PAPP-A } \\
\text { (MoM) }\end{array}$ & $\begin{array}{l}\text { CRL } \\
(\mathrm{mm})\end{array}$ & $\begin{array}{l}\text { TN } \\
(\mathrm{mm})\end{array}$ & $\begin{array}{l}\mathrm{CT} \\
\text { riesgo } \\
(1:)\end{array}$ & Ecografía & Cariotipo & Evolución \\
\hline 1 & 33 & 563 & 0,56 & 0,32 & 48 & 5,1 & 50 & & $45 \times 0$ & IVE \\
\hline 2 & 41 & 48 & 4,39 & 0,41 & 55 & 3,5 & 50 & & 47XXT21 & IVE \\
\hline 3 & 38 & 97 & 1,61 & 0,39 & 72 & 1,0 & 131 & & 47XYT21 & IVE \\
\hline 4 & 33 & 326 & 2,99 & 0,18 & 51 & 1,1 & 50 & & 47XYT21 & IVE \\
\hline 5 & 30 & 476 & 1,54 & 0,90 & 70 & 8,0 & 50 & & $45 \times 0$ & IVE \\
\hline 6 & 32 & 412 & 1,27 & 0,13 & 58 & 1,2 & 142 & & $47 X X T 21$ & IVE \\
\hline 7 & 32 & 412 & 0,30 & 0,66 & 45 & 4,3 & $\begin{array}{l}50 \text { (T21) } \\
50 \text { (T18) }\end{array}$ & PMF & 47XXT18 & IVE \\
\hline 8 & 28 & 685 & 0,29 & 0,50 & 48 & 3,3 & $\begin{array}{l}50 \text { (T21) } \\
50 \text { (T18) }\end{array}$ & & 47XYT18 & IVE \\
\hline 9 & 39 & 75 & 0,24 & 0,38 & 56 & 2,6 & 113 & & 47XYT21 & IVE \\
\hline 10 & 27 & 679 & 1,06 & 0,30 & 62 & 4,4 & 50 & & 47XYT21 & IVE \\
\hline 11 & 30 & 510 & 1,09 & 0,60 & 57 & 1,0 & 4354 & PMF & $69 X X X$ & IVE \\
\hline 12 & 19 & 1099 & 1,77 & 0,46 & 63 & 2,4 & 214 & & 47XYT21 & IVE \\
\hline 13 & 39 & 75 & 0,33 & 0,74 & 43 & 2,6 & $\begin{array}{l}114(\mathrm{~T} 21) \\
50(\mathrm{~T} 18)\end{array}$ & & 47XYT18 & IVE \\
\hline 14 & 42 & 39 & 0,95 & 0,65 & 53 & 1,5 & 362 & CAV & 47XYT21 & IVE \\
\hline 15 & 37 & 143 & 2,02 & 0,57 & 69 & 3,1 & 50 & & $47 X X T 21$ & IVE \\
\hline 16 & 33 & 335 & 5,44 & 0,89 & 62 & 1,9 & 109 & & $47 X X T 21$ & IVE \\
\hline 17 & 33 & 376 & 1,21 & 0,36 & 59 & 3,3 & 50 & & $47 X X T 21$ & IVE \\
\hline 18 & 39 & 86 & 1,43 & 1,12 & 67 & 6,0 & 50 & & 47XXT21 & IVE \\
\hline 19 & 19 & 1010 & 1,60 & 0,25 & 60 & 3,0 & 50 & & 47XYT21 & IVE \\
\hline 20 & 35 & 196 & 2,28 & 0,29 & 54 & 1,6 & 50 & & 47XYT21 & IVE \\
\hline 21 & 43 & 25 & 2,01 & 0,31 & 63 & 2,6 & 50 & & 47XYT21 & IVE \\
\hline 22 & 17 & 961 & 2,64 & 0,25 & 54 & 3,5 & 50 & & $47 X X T 21$ & IVE \\
\hline 23 & 41 & 47 & 1,78 & 0,20 & 58 & 2,0 & 50 & & 47XXT21 & IVE \\
\hline 24 & 24 & 1108 & 4,38 & & & & & $\mathrm{DW}+\mathrm{HVI}$ & 47XYT13 & IVE \\
\hline 25 & 37 & 136 & 1,69 & 1,41 & 45 & 0,8 & 2405 & $\mathrm{VMC}+\mathrm{CIV}$ & 47XYT21 & IVE \\
\hline 26 & 30 & 588 & 0,97 & 0,10 & 61 & 1,30 & 347 & & 47XYT21 & IVE \\
\hline 27 & 35 & 240 & 4,80 & 0,25 & 62 & 3,2 & 50 & & 47XYT21 & IVE \\
\hline 28 & 35 & 205 & 1,84 & 0,21 & 53 & 1,1 & 50 & & $47 X X T 21$ & IVE \\
\hline 29 & 36 & 176 & 1,28 & 0,35 & 47 & 2,2 & 50 & & 47XYT21 & IVE \\
\hline 30 & 36 & 194 & 2,95 & 0,52 & 75 & 2,7 & 50 & & 47XXT21 & IVE \\
\hline 31 & 32 & 387 & 1,29 & 0,55 & 65 & 0,8 & 2001 & CAV & 47XXT21 & IVE \\
\hline 32 & 31 & 491 & 0,95 & 0,35 & 39 & 1,4 & 522 & & $47 X X T 21$ & $\begin{array}{l}\text { Normal a los } 6 \\
\text { meses. Ecocar- } \\
\text { dio postnatal: } \\
\text { CIA }\end{array}$ \\
\hline 33 & 45 & 20 & & & & & & & 47XYT21 & $\begin{array}{l}\text { Normal a los } 6 \\
\text { meses }\end{array}$ \\
\hline 34 & 34 & 265 & 1,02 & 0,38 & 55 & 1,3 & 788 & & $47 X Y T 21$ & $\begin{array}{l}\text { Normal a los } 6 \\
\text { meses. Ecocar- } \\
\text { dio posnatal: CIA }\end{array}$ \\
\hline 35 & 32 & 412 & & & & & & & 47XYT21 & $\begin{array}{l}\text { Normal a los } 6 \\
\text { meses }\end{array}$ \\
\hline
\end{tabular}

CT : Test combinado. TN: Translucencia nucal. CRL: longitud céfalo-caudal PMF: Polimarformado. CAV: Canal atrioventricular. CIV: Comunicación interventricular. VMC: Ventriculomegalia cerebral. DW+HVI: Dandy Walker+hipoplasia ventricular izquierda. CIA: Comunicación interauricular. IVE : Interrupción voluntaria del embarazo. PAPP-A: Pregnancyassociated plasma protein A. 
Tabla IV

PREVALENCIA Y TASA DE DIAGNÓSTICO PRENATAL ECOGRÁFICO DE LAS MALFORMACIONES ESTRUCTURALES PRESENTADA POR SISTEMAS ANATÓMICOS

\begin{tabular}{|c|c|c|c|c|c|}
\hline & & & & $\begin{array}{l}\text { Malformación } \\
\text { mayor }\end{array}$ & $\begin{array}{l}\text { Malformación } \\
\text { menor }\end{array}$ \\
\hline Sistema anatómico & $\mathrm{n}$ & $\begin{array}{c}\text { Prevalencia } \\
(\%)\end{array}$ & $\begin{array}{c}\text { Sensibilidad } \\
(\%)\end{array}$ & $\begin{array}{c}\text { Sensibilidad } \\
(\%)\end{array}$ & $\begin{array}{c}\text { Sensibilidad } \\
(\%)\end{array}$ \\
\hline Sistema cardiovascular & 70 & 0,56 & 52,85 & $92,59(25 / 27)$ & $27,90(12 / 43)$ \\
\hline Sistema nervioso central & 39 & 0,31 & 84,61 & $95,93(20 / 21)$ & $72,22(13 / 18)$ \\
\hline Tracto urogenital & 67 & 0,53 & 100,0 & $100(27 / 27)$ & $100(40 / 40)$ \\
\hline $\begin{array}{l}\text { Tracto gestrointestinal y pared } \\
\text { abdominal }\end{array}$ & 28 & 0,22 & 82,14 & $100(15 / 15)$ & $61,5(8 / 13)$ \\
\hline Sistema músculo esquelético & 35 & 0,28 & 88,57 & $88,8(16 / 18)$ & $88,23(15 / 17)$ \\
\hline Sistema respiratorio & 8 & 0,06 & 100,0 & $100(7 / 7)$ & $100(1 / 1)$ \\
\hline Total & 247 & 1,97 & 80,56 & $95,65(110 / 115)$ & $67,42(89 / 132)$ \\
\hline
\end{tabular}

Al nacimiento la prevalencia de malformaciones estructurales fue de 1,5\% [IC 95\%: 1,3-1,7](200 casos). Hubo un 0,3\% [IC 95\%: 0,2-0,4](42 casos) de malformaciones estructurales mayores, de las que $34(0,2 \%)$ fueron diagnosticados mediante una ecografía antes del nacimiento. En 8 casos ( 1 caso de ventriculomegalia, 1 caso de tetralogía de Fallot, 1 caso de canal auriculoventricular parcial, 1 caso de estenosis pulmonar, 2 casos de labio leporino y 2 casos de deformidades importantes de miembros) $(0,06 \%$ [IC 95\%: $0,02-0,1])$ no se consiguió un diagnóstico prenatal ecográfico.

Durante el tiempo analizado se han realizado 714 técnicas invasivas: 504 amniocentesis (70,5\%), 185 biopsias coriales $(25,8 \%)$ y 25 cordocentesis $(3,6 \%)$. Al $6,04 \%$ de las gestantes se les ha practicado alguna técnica invasiva. No se observó ninguna pérdida gestacional imputable a la técnica (2 pérdidas gestacionales no imputables a la técnica). No se ha notificado de ningún caso de fallo de crecimiento de la muestra. Las indicaciones y el tipo de técnica invasiva han ido cambiando a lo largo de los semestres como se observa en la Tabla VI.

\section{DISCUSIÓN}

La prevalencia de defectos congénitos en nuestra población $(2,26 \%)$ es similar a lo descrito por los diferentes registros de malformaciones congénitas para poblaciones de bajo riesgo $(1,15)$. Las malformaciones más prevalentes en nuestra población han sido las cardiacas seguidas de las renales y las del sistema nervioso central, estos datos coinciden con otros registros de malformaciones congénitas $(1,15)$.

En la literatura internacional existen tasas de detección de malformaciones estructurales muy variables, entre un 13,3\%-82,4\% (1,3,4,7,16-21). El grupo EUROCAT (21) presenta una tasa de detectación para malformaciones estructurales del $25 \%$, variando entre 8 y $79 \%$ dependiendo del área geográfica. Este grupo presenta una tasa de detección para las malformaciones estructurales mayores del $68 \%$ (22). En este grupo la malformación más frecuentemente diagnosticada ha sido la anencefalia $(94 \%)$ y la menos diagnosticada ha sido la transposición de grandes arterias (24\%) (22). En general, se considera que la sensibilidad de la ecografía para la detección de malformaciones estructurales es de un $40 \%$ (7). Según Gonçalves (7) y el grupo EUROCAT (22), la sensibilidad de la mínima aceptable de la ecografía, de los centros que ofrecen diagnóstico prenatal, para la detección de malformaciones estructurales mayores debe ser de un $60-80 \%$. Consideramos que nuestra tasa de detección de malformaciones estructurales es adecuada ya que se han detectado ecográficamente el $95 \%$ de las mayores lo que lleva a una prevalencia al nacimiento de un $0,34 \%$ y sólo el $0,06 \%$ de estas no ha sido posible su diagnóstico ecográfico prenatal.

Según nuestros resultados las malformaciones renales, respiratorias y del sistema nervioso central son las más frecuentemente diagnosticadas, y las más difíciles de diagnosticar prenatalmente son las cardiacas. Diferentes autores encuentran igualmente dificultad en el diagnóstico de las malformaciones fetales $(3,21,23-25)$. Un dato positivo en nuestro trabajo es la alta sensibilidad de la ecografía para la detección de malformaciones cardíacas mayores $(85,7 \%)$, lo que permite crear pautas de actuación prenatal y neonatal para aquellos casos afectados. 
Tabla V

\section{DESCRIPCIÓN DE MALFORMACIONES ESTRUCTURALES POR SISTEMA ANATÓMICO Y TASA DE DETECCIÓN}

\begin{tabular}{|c|c|c|c|}
\hline Sistema anatómico & $\begin{array}{l}\text { Total } \\
\text { n (\%) }\end{array}$ & Diagnosticados & $\begin{array}{c}\text { No } \\
\text { diagnosticados }\end{array}$ \\
\hline Sistema nervioso central & $39(15,78 \%)$ & 33 & 6 \\
\hline Anencéfalo & 7 & 7 & - \\
\hline Hidrocefalia & 5 & 4 & 1 \\
\hline Espina bífida & 8 & 8 & - \\
\hline Defecto de fosa posterior & 11 & 11 & - \\
\hline Microcefalia & 3 & - & 3 \\
\hline Otros & 4 & 2 & 2 \\
\hline Sistema cardiovascular & $70(28,34 \%)$ & 37 & 33 \\
\hline Defecto del tabique ventricular & 26 & 6 & 20 \\
\hline Defecto del tabique auricular & 5 & - & 5 \\
\hline Tetralogía de Fallot & 4 & 3 & 1 \\
\hline Transposición de grandes arterias & 1 & 1 & - \\
\hline Truncus arterioso & 3 & 3 & - \\
\hline Canal atrioventricular & 6 & 5 & 1 \\
\hline Hipoplasia ventricular & 6 & 6 & - \\
\hline Coartación de aorta & 3 & 3 & - \\
\hline Anomalía cardiaca compleja & 4 & 4 & - \\
\hline Otros & 12 & 6 & 6 \\
\hline Tracto urogenital & $67(27,12 \%)$ & 67 & 0 \\
\hline Agenesia renal uni/bi & 12 & 12 & - \\
\hline Displasia renal uni/bi & 11 & 11 & - \\
\hline Uropatía obstructiva & 26 & 26 & - \\
\hline Megavejiga & 4 & 4 & - \\
\hline Quiste anexial & 3 & 3 & - \\
\hline Otros & 8 & 8 & - \\
\hline Tracto gastrointetinal y defecto de pared abdominal & $28(11,33 \%)$ & 23 & 5 \\
\hline Obstrucción & 4 & 3 & 1 \\
\hline Atresia duodenal & 0 & - & - \\
\hline Atresia esofágica & 3 & 2 & 1 \\
\hline Atresia biliar & 1 & - & 1 \\
\hline Ano imperforado & 3 & 1 & 2 \\
\hline Hernia diafragmática & 7 & 7 & - \\
\hline Onfalocele & 4 & 4 & - \\
\hline Gastrosquisis & 3 & 3 & - \\
\hline Hernia umbilical & 1 & 1 & - \\
\hline Otros & 2 & 2 & - \\
\hline Músculo esquelética & $35(14,17 \%)$ & 31 & 4 \\
\hline Deformidad de miembro & 9 & 7 & 2 \\
\hline Reducción de miembro & 6 & 6 & - \\
\hline Displasia esquelética & 4 & 4 & - \\
\hline Defecto del labio +/- paladar & 8 & 6 & 2 \\
\hline Otros & 8 & 8 & - \\
\hline Sistema respiratorio & $8(3,27 \%)$ & 8 & 0 \\
\hline Quiste pulmonar & 7 & 7 & - \\
\hline Secuestro pulmonar & 1 & 1 & - \\
\hline TOTAL & 247 (100\%) & $199(80,56 \%)$ & $48(19,44 \%)$ \\
\hline
\end{tabular}




\section{Tabla VI}

\section{MOTIVOS DE LA REALIZACIÓN DE TÉCNICAS INVASIVAS PARA EL DIAGNÓSTICO PRENATAL}

\begin{tabular}{lrrrrrr}
\hline Motivo & \multicolumn{1}{c}{$2^{\circ} \mathrm{S}-2006$} & 1er S-2007 & $2^{\circ} \mathrm{S}-2007$ & 1er S-2008 & $2^{\circ}$ S-2008/09 & Total \\
\hline Edad materna & $15,7 \%(32)$ & $11,8 \%(14)$ & $11,2 \%(11)$ & $7,3 \%(9)$ & $4,6 \%(8)$ & $10,3 \%(74)$ \\
$\begin{array}{l}\geq 35 \text { años) } \\
\text { Angustia materna }\end{array}$ & $4,4 \%(9)$ & $6,7 \%(8)$ & $4,1 \%(4)$ & $6,5 \%(8)$ & $2,3 \%(4)$ & $4,6 \%(33)$ \\
Sospecha ecográfica & $5,4 \%(11)$ & $19,3 \%(23)$ & $19,4 \%(19)$ & $7,3 \%(9)$ & $8,8 \%(15)$ & $10,7 \%(77)$ \\
CT & $62,7 \%(128)$ & $50,4 \%(60)$ & $60,2 \%(59)$ & $69,4 \%(86)$ & $75,4 \%(129)$ & $64,7 \%(462)$ \\
DT & $6,9 \%(14)$ & $5,0 \%(6)$ & $3,1 \%(3)$ & $4,0 \%(5)$ & $5,8 \%(10)$ & $5,3 \%(38)$ \\
Otros & $4,9 \%(10)$ & $6,7 \%(8)$ & $2,1 \%(2)$ & $5,6 \%(7)$ & $1,8 \%(3)$ & $4,2 \%(30)$ \\
Total & 204 & 119 & 98 & 124 & 169 & $100 \%(714)$ \\
\hline
\end{tabular}

CT: Test combinado. DT: Doble Test. S: Semestre.

La prevalencia de cromosomopatías es de un $0,28 \%$ (el $80 \%$ son síndromes de Down), lo que nos indica que es una población de bajo riesgo para cromosomopatías. Observamos que el $57,1 \%$ de los fetos afectos de síndrome de Down se encuentran en mujeres con edad menor de 35 años; por lo que un cribado de cromosomopatías basado solo en la edad materna no permitiría detectar en nuestra serie más de la mitad de los casos de síndrome de Down.

Hemos conseguido implantar un cribado de cromosomopatías de forma universal (ofertado al $95,1 \%$ de las gestantes). La sensibilidad del test combinado para el síndrome de Down es del 76,9\% (tasa de falsos positivos del 4,3\%). Nuestros datos son comparables a los de la bibliografía internacional $(5,6)$. Podemos afirmar que hemos instaurado un cribado de cromosomopatías de garantías basado en un test combinado y como la prevalencia al nacimiento del síndrome de Down ha disminuido al $0,03 \%$ (3 casos por cada 10.000 nacimientos) podemos decir que este cribado es eficaz para el diagnóstico prenatal de cromosomopatías.

Al 6,0\% de nuestras gestantes se les realizó una técnica invasiva. Se observa como las gestantes mayores de 35 años han optado por realizarse un cribado de cromosomopatías en vez de una técnica invasiva directa. Ha sido necesaria 25 técnicas invasivas por caso de cromosomopatía diagnosticada, cifra que se considera adecuada tras aplicar un método de cribado (22).

La prevalencia de defecto congénito ha descendido de un $2,2 \%$ a un $1,6 \%$ al nacimiento $(0,4 \%$ sin diagnosticar) tras la implantación del cribado de cromosomopatías y de malformaciones estructurales. De los recién nacidos afectos de anomalía con- génita al nacimiento, 4 tenían una cromosomopatía y 8 una malformación mayor. Esto supone que solo un $0,09 \%$ de los recién nacidos están afectos al nacimiento de un defecto congénito mayor.

Con las limitación de la recogida de los datos, de sólo 3 días tras el nacimiento, hemos observados que la prevalencia de los defectos congénitos no diagnosticados al nacimiento disminuyó hasta el $0,4 \%$, y el de los defectos congénitos mayores disminuyó al 0,09\% tras la aplicación de un cribado universal y de garantías de malformaciones estructurales y de anomalías cromosómicas fetales.

\section{CONCLUSIÓN}

Se ha instaurado un cribado de defectos congénitos que ha logrado disminuir la prevalencia de los defectos congénitos al nacimiento sin diagnosticar a un $0,4 \%$ y la de los defectos congénitos mayores a un $0,09 \%$.

\section{BIBLIOGRAFÍA}

1. The status of health in the European Union: congenital malformations. EUROCAT Central Registry, University of Ulster. Available at: www.eurocat.ulster.ac.uk/ $\mathrm{pdf} /$.special-report-con-malfs.pdf.

2. Kaback MM. The utility of prenatal diagnosis. In Rodeck $\mathrm{CH}$ \& Nicolaides $\mathrm{KH}$ (eds). Prenatal Diagnosis. New York: John Wiley. 1984:53-64.

3. Levi S. Ultrasound in prenatal diagnosis: polemics around routine ultrasound screening for second trimester foetal malformations. Prenat Diagn 2002;22:285-95.

4. Grandjean H, Larroque D, Levi S, and the Eurofetus team. The performance of routine ultrasonographic screening of pregnancies in the Eurofetus study. Am J Obstet Gynecol 1999;181:446-54.

5. Wald NJ, Rodeck C, Hackshaw AK, Walters J, Chitty 
L, Mackinson AM; SURUSS Research Group. First and second trimester antenatal screening for Down's syndrome: the results of the Serum, Urine and Ultrasound Screening Study (SURUSS). Health Technol Assess 2003;7:1-77.

6. Malone FD, Canick JA, Ball RH, Nyberg DA, Comstock $\mathrm{CH}$, Bukowski R, et al. First-trimester or secondtrimester screening, or both, for Down's syndrome. N Engl J Med 2005;353:2001-11.

7. Gonçalves LF. Diagnóstico prenatal de malformaciones anatómicas congénitas. In: Sonograhy in Obstetrics and Gynecology, 6th ed. The McGraw-Hill Companies, New York, NY 10020. 2002:341-73.

8. Troyano J, Usandizaga M, Escurrida M, Valero J, Montalvo J, Martínez-Cortés L, et al. Organización de la ecografía obstétrico-ginecológica. Recomendaciones para la organización de un Servicio de Obstetricia y Ginecología. Documentos de la Sociedad Española de Ginecología y Obstetricia. Madrid: Sociedad Española de Ginecología y Obstetricia 31-40, 2005.

9. Royal College of Obstetricians and Gynaecologists. Routine Ultrasound Screening in Pregnancy: Supplement to Ultrasound Screening for Foetal Abnormalities. London:RCOG Press. 2000.

10. Nicolaides KH. The $11-13+6$ weeks scan. Foetal Medicine Foundation. London 2004.

11. Spencer K, Souter V, Tul N, Snijders R, Nicolaides $\mathrm{KH}$. A screening program for trisomy 21 at $10-14$ weeks using fetal nuchal translucency, maternal serum free ß-human chorionic gonadotropin and pregnancyassociated plasma protein-A. Ultrasound Obstet Gynecol 1999;13:231-7.

12. Tul N, Spencer K, Noble P, Chan C, Nicolaides KH. Screening for trisomy 18 by fetal nuchal translucency and maternal serum free B-hCG B-human chorionic gonadotropin and pregnancy-associated plasma protein and PAPP-A at 10-14 weeks of gestation. Prenat Diagn 1999;19:1035-42.

13. Reynolds TM, Penney MD. The mathematical basis of multivariate risk screening: with special reference to screening for Down's syndrome associated pregnancy. Ann Clin Biochem 1990;27(PT5):452-8.

14. Armitage P. Estadística para la Investigación Biomédica. Harcout Brace 1997.

15. Martínez-Frías, Bermejo E y Grupo Periférico del ECEMC. Anomalías Congénitas en España. Análisis clínico de los recién nacidos con defectos congénitos registrados en el ECEMC: distribución por etiología y por grupos étnicos. Bol ECEMC Rev Dismor Epidemiol 2009;8:24-47.

16. Skupski DW, Newman S, Edersheim T, Hutson JM, Udom-Rice I, Chervenak FA, McCullough LB. The impact of routine obstetric ultrasonographic screening in a low-risk population. Am J Obstet Gynecol 1996;175:1142-5.

17. Romosan G, Henriksson E, Rylander A, Valentin L. Diagnostic performance of routine ultrasound screening for fetal abnormalities in an unselected Swedish population in 2000-2005. Ultrasound Obstet Gynecol 2009;34:526-33.

18. Crane JP, LeFevre ML, Winborn RC, Evans JK, Ewigman BG, Bain RP, et al. A randomized trial of prenatal ultrasonographic screening: impact on the detection, management, and outcome of anomalous fetuses. The RADIUS Study Group. Am J Obstet Gynecol 1994;171:392-9.

19. VanDorsten J, Hulsey TC, Newman RB, Menard MK. Fetal anomaly detection by second-trimester ultrasonography in a terciary center. Am J Obstet Gynecol 1998;178:742-9.

20. Carrera JM, Torrents M, Mortera C, Cusi V, Munoz A. Routine prenatal ultrasound screening for fetal abnormalities: 22 years'experience. Ultrasound Obstet Gynecol 1995;5:174-9.

21. European Surveillance of Congenital Anomalies (EUROCAT). Available at: http://www.eurocat.ulster. ac.uk.

22. Garne E, Loane M, Dolk H, De Vigan C, Scarano G, Tucker D, et al. Prenatal diagnosis of severe structural congenital malformations in Europe. Ultrasound Obstet Gynecol 2005;25:6-11.

23. Ott WJ. The accuracy of antenatal fetal echocardiography screening in high- and low-risk patients. Am J Obstet Gynecol. 1995;172:1741-7.

24. Tegnander E, Willians W, Johansens OJ, Blaas K, Eik$\mathrm{Nes} \mathrm{H}$. Prenatal detection of heart defects in a non-selected population of 30149 fetuses-detection rates and outcome. Ultrasound Obstet Gynecol 2006;27:252-65.

25. Chew C, Halliday JL, Riley M, Penny DJ. Populationbased study of antenatal detection of congenital heart disease by ultrasound examination. Ultrasound Obstet Gynecol 2007;29:619-24. 